\title{
Path integral of the relativistic point particle in Minkowski space
}

\author{
Benjamin Koch $\circledast^{1,2, *}$ and Enrique Muñoz $\odot^{2, \dagger}$ \\ ${ }^{1}$ Institut für Theoretische Physik, Technische Universität Wien, \\ Wiedner Hauptstrasse 8-10, A-1040 Vienna, Austria \\ ${ }^{2}$ Pontificia Universidad Católica de Chile, Instituto de Física, Pontificia Universidad Católica de Chile, \\ Casilla 306, Santiago, Chile
}

(Received 23 February 2021; accepted 15 April 2021; published 28 May 2021)

\begin{abstract}
In this article, we analyze the fundamental global and local symmetries involved in the action for the free relativistic point particle in Minkowski space. Moreover, we identify a hidden local symmetry, whose explicit consideration and factorization utilizing of a Fujikawa prescription leads to the construction of relativistic two-point correlation functions that satisfy the Chapman-Kolmogorov identity. By means of a detailed topological analysis, we find three different relativistic correlation functions (orthochronous, spacelike, and Feynman) which are obtained from the exclusive integration of paths within different sectors in Minkowski space. Finally, the connection of this approach to the Feynman checkerboard construction is explored.
\end{abstract}

DOI: $10.1103 /$ PhysRevD.103.105025

\section{INTRODUCTION}

\section{A. The context}

Symmetries are key ingredients of our construction recipes for fundamental field theories. Local symmetries (gauge symmetries) give us a method to study interactions, while global symmetries give us a way to describe conserved charges via the Noether theorem. For this article, two cases will be particularly important. The first case is global Lorentz invariance, which leads to conserved quantities such as mass, momentum, angular momentum, and center-of-mass energy. This symmetry is an essential part of all fundamental field theories, and it is intimately linked to the spin-statistics relation of fundamental particles. The second case is a gauge symmetry known as diffeomorphism invariance, which is, for example, the underlying symmetry of the theory of general relativity (GR).

Another indispensable ingredient of any fundamental physical theory is that it needs to be quantized. While the combination of global Lorentz invariance with the principles of quantum mechanics works out nicely, a consistent quantization of general relativity is still an open problem. It is widely believed that this problem is somehow linked to diffeomorphism invariance as the underlying gauge

\footnotetext{
*benjamin.koch@tuwien.ac.at

†munozt@fis.puc.cl
}

Published by the American Physical Society under the terms of the Creative Commons Attribution 4.0 International license. Further distribution of this work must maintain attribution to the author(s) and the published article's title, journal citation, and DOI. Funded by SCOAP ${ }^{3}$. symmetry of GR. Unfortunately, gravity in $(1+3)$ dimensions is a very complicated theory with this symmetry. Thus, the community has put a large effort into studying and understanding somewhat simpler theoretical realizations of this symmetry, such as, for example, Euclidean quantum gravity [1-4] or quantum gravity with a Lorentz signature but in lower-dimensional systems [5-7]. The most simple theoretical system with diffeomorphism invariance is the action of the relativistic point particle. Attempts to quantize the straight action of the relativistic point particle (1) lead to substantial difficulties [8-14], such as the loss of the Chapman-Kolmogorov identity. In the literature, these problems are circumvented by using a different, but classically equivalent, action $[11,15,16]$, by introducing a modified theory of probabilities [17-19], by the restriction to particular manifolds [20], by the use of approximations [14], or by a description based on a subtle limit of a quantum field theoretical prescription [21].

In a series of previous papers, we have shown that these problems with the quantization of the action (1) can actually be solved by taking into consideration yet another (hidden) local symmetry, corresponding the invariance of the square of the 4-velocity $\left(d x^{\mu} / d \lambda\right)\left(d x_{\mu} / d \lambda\right)$ under Lorentz boosts and rotations. Despite this not being a gauge symmetry, we have shown from three different and complementary approaches that it can nevertheless be factored out in the path-integral (PI) construction, thus restoring the Chapman-Kolmogorov property in the resulting two-point correlation functions (TPCFs). We developed this new method in three complementary PI approaches:

(i) a PI over the Hamiltonian action and the corresponding constraint analysis [22]; 
(ii) a direct PI in Euclidean space [23]; and

(iii) a formal functional path integral [24].

These results were obtained either in Euclidean space or with abstract formal methods, but a detailed analysis of the much richer structure of Minkowski space is missing.

This article closes this gap by discussing the path integral quantization of the relativistic point particle in Minkowski space-time, while paying explicit attention to the causal structure of virtual paths. By doing so, new insights can be obtained on how different types of paths lead to different TPCFs. Such a discussion is particularly interesting, since it gives evidence that also in quantum gravity the path integral quantization will give results that are sensitive to the causal structure of the system.

This article is organized as follows. In the next two subsections of the introduction, we will introduce the relativistic point particle action and the conceptual steps which have to be considered in the quantization of this system. The notation of this introduction closely follows Ref. [23]. In the next section, the quantization procedure will be realized. First, the discrete action will be defined, and then the two-step TPCF will be calculated before this result is generalized to the $N+1$ step TPCF. This discussion will be done by summing exclusively over causal timelike (orthochronous) paths. A generalization with a comparison to spacelike and timelike noncausal paths follows in the next subsection. Then, a particular case of this calculation is considered, where a spatial flip symmetry is removed from the construction. This results in the famous Feynman checkerboard. After generalizing the TPCFs with flip symmetry to $1+d$ dimensions and commenting on the higher-dimensional checkerboard, we summarize our findings in the conclusions.

\section{B. The relativistic point particle}

The action for a relativistic point particle in Minkowski space with the metric signature $g^{00}=+1, g^{i i}=-1$ for $i=1,2,3$ is

$$
S=-\int_{\lambda_{i}}^{\lambda_{f}} d \lambda \cdot m \sqrt{\left(\frac{d x^{\mu}}{d \lambda} \frac{d x_{\mu}}{d \lambda}\right)},
$$

where $x^{\mu}\left(\lambda_{i}\right)=x_{i}^{\mu}$ and $x^{\mu}\left(\lambda_{f}\right)=x_{f}^{\mu}$. This is simply the mass $(m)$ times the geometric length of a given path $\mathcal{P}$ in Euclidean space or, equivalently, the mass times the total interval between two events in Minkowski space. This action and its corresponding Lagrangian are equipped with several symmetries which will be important for the formulation of a consistent path integral $[12,13]$.

(a) Global Poincaré invariance. - This can be seen from the fact that the action is invariant under global rotations, boosts, and shifts of the coordinate system.

(b) Local Lorentz invariance.-This means that the Lagrangian is invariant under local rotations and boosts of the vector $\left(d x^{\mu}\right) /(d \lambda)$ at any point along the trajectory. Since this symmetry vanishes at the classical level and, thus, its role on the construction of the TPCFs is typically neglected in the literature, we termed it a "hidden symmetry." We presented a formal argument on why this symmetry, which is not a classical gauge symmetry, is important in this given context in Refs. [22-24]. In Ref. [22], we further showed in the Hamiltonian action formulation that there is a nontrivial constraint associated with this symmetry.

(c) Weyl invariance.-This means that the Lagrangian does not depend on the way that $\lambda$ parametrizes a path $\mathcal{P}$. The change to any other monotonic function $\tilde{\lambda}(\lambda)$ would leave the Lagrangian invariant.

In the following, the symmetry (a) will be used to choose the coordinate system such that $x_{i}^{\mu}=0$ and that $x_{f}^{\mu}$ is different from zero in only one component. The symmetries (b) and (c) are symmetries which have to be treated with care when it comes to realizing an integral over different paths, since two seemingly different paths could be actually physically equivalent. The overcounting of physically equivalent paths would result in a wrong weight of some paths with respect to others.

\section{General considerations on the explicit form of the PI measure}

A naive, straightforward definition of a path integral for the relativistic point particle fails to satisfy the Kolmogorov condition for transition probability amplitudes. As shown in Refs. [22-24], this is due to the overcounting arising from the symmetries (b), and (c), that needs to be properly factored out either by geometric considerations [22,23] or by a group theoretical analysis involving the Fadeev-Popov method $[24,25]$.

Following the geometric approach, let us consider as an example the case of two intermediate points $n=2$. All configurations where the position $x_{1}^{\mu}$ is on the classical path between $x_{i}^{\mu} \rightarrow x_{2}^{\mu}$ correspond actually to the same path $x_{i}^{\mu} \rightarrow x_{2}^{\mu} \rightarrow x_{f}^{\mu}$ as shown in Fig. 1. Strictly speaking, they should be counted only once, but according to a naive counting this path would be counted multiple times. In many cases, this type of overcounting is not relevant, since the number of paths that are not overcounted grows much

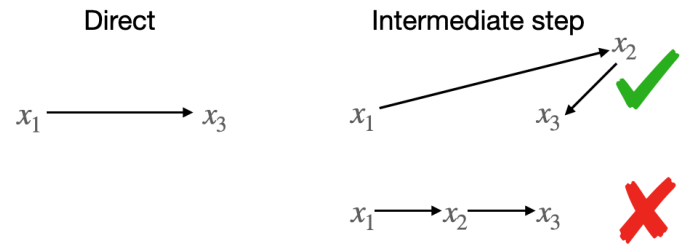

FIG. 1. Exemplification of possible overcounting of one path with two intermediate steps, which is already counted in the PI with one intermediate step. 
faster with $n$ and $D$ than the number of paths where this overcounting occurs. Thus, an improved definition of the path integral can be given by

$$
\begin{aligned}
K\left(x_{i}^{\mu}, x_{f}^{\mu}\right) & \left.\equiv \lim _{n \rightarrow \infty} \sum_{j=1}^{n} K^{(j)}\left(x_{i}^{\mu}, x_{f}^{\mu}\right)\right|_{\mathrm{NOC}} \\
& \equiv \lim _{n \rightarrow \infty} \sum_{j=1}^{n}\left(\left.\prod_{l=1}^{j} \int d x_{l}^{D}\right|_{\mathrm{NOC}}\right) \Xi_{j} \exp \left[-i S_{j}\right] \\
& =\left.\int d x_{1}^{D}\right|_{\mathrm{NOC}} \Xi_{1} \exp \left[-i S_{1}\right] \\
& +\left.\int d x_{1}^{D} \int d x_{2}^{D}\right|_{\mathrm{NOC}} \Xi_{2} \exp \left[-i S_{2}\right]+\cdots,
\end{aligned}
$$

where $\left.\right|_{\text {NOC }}$ stands "integrate and sum without overcounting" in the sense of the symmetries described in (b) and (c). Furthermore, $\Xi_{i}$ is the Fujikawa determinants assuring the invariance of the measure under these symmetries [26]. The action for a path with $q$ intermediate steps (defining $\left.x_{j=0}^{\mu}=x_{i}^{\mu}, x_{j=q+1}^{\mu}=x_{f}^{\mu}\right)$ is given by the recursive relation

$$
S_{q}=-m \sum_{j=1}^{q+1} \sqrt{\left(x_{j}-x_{j-1}\right)^{\mu}\left(x_{j}-x_{j-1}\right)_{\mu}} .
$$

Relation (2) will be used for the calculation of the path integral of the relativistic point particle in Minkowski space. The following discussion will be done in $D=1+1$ dimensions, and a generalization to an arbitrary number of dimensions will be given in Sec. II G.

\section{THE TPCF}

We shall first consider a geometrical analysis of the problem, closely following Ref. [23], starting with the calculation of the one-slice TPCF and then generalizing to the $n$-slice TPCF.

\section{A. Action}

For the one-slice TPCF, based on the global Poincare invariance (a), we can choose $x_{i}^{\mu}=(0,0)$, going to $x_{f}^{\mu}=$ $\left(t_{f}, 0\right)$ via $x_{1}^{\mu}=\left(t_{1}, x_{1}\right)$. Since the action of the relativistic point particle does not depend on the sign of $t_{f}$, we will work with purely positive time differences $t_{f}=\left|t_{f}\right|$. The action for this configuration is (see Fig. 2)

$$
\begin{aligned}
S=S_{1}= & -m\left(\sqrt{\left(x_{f}-x_{1}\right)_{\mu}\left(x_{f}-x_{1}\right)^{\mu}}\right. \\
& \left.+\sqrt{\left(x_{1}-x_{i}\right)_{\mu}\left(x_{1}-x_{i}\right)^{\mu}}\right) .
\end{aligned}
$$

The minimum for (4) is obtained for the classical motion along a straight line:
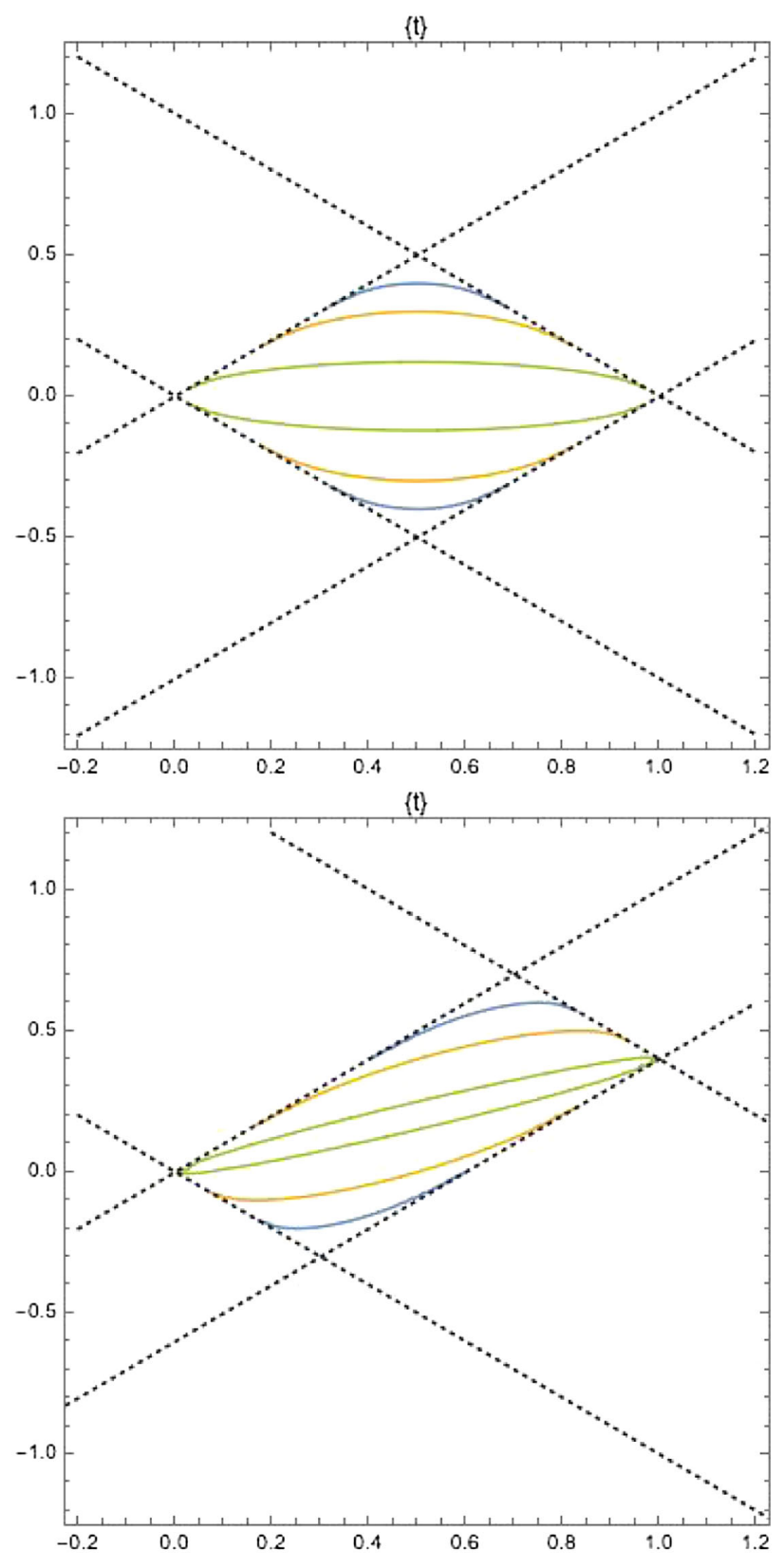

FIG. 2. Contours of constant action (4). The light cones of the initial and final point are black dotted lines. The upper figure is for $x_{f}=(1,0)$, and the lower figure is for $x_{f}=(1,0.4)$. For both figures, $x_{i}(0,0)$ and $m=1$ were chosen.

$$
S_{c l}=-m \sqrt{\left(x_{f}-x_{i}\right)_{\mu}\left(x_{f}-x_{i}\right)^{\mu}} .
$$

When considering only orthochronous paths which lie in the timelike future light cone of $x_{i}$ and the timelike past light cone of $x_{f}$, the maximum value for (4) is zero. In most of the following discussions, we will expect the TPCFs to be Lorentz invariant. In those cases, one can, without loss of generality, choose a reference system where $x_{i}^{\mu}=(0,0)$ and $x_{f}^{\mu}=\left(t_{f}, 0\right)$. 


\section{B. The two-step TPCF}

With the action (4), one can define the TPCF with one intermediate integration point:

$$
K^{(1)}=\mathcal{N} \cdot \iint_{V} d t_{1} d x_{1} \Xi_{1} e^{+i S_{1}},
$$

where the integration volume $V$ is given by the overlap of the future light cone of $x_{i}$ with the past light cone of $x_{f}$. Note that for all "reasonable" paths the action (1) is negative, which is why the exponential in (6) was defined with a plus sign instead of a minus sign. The term $\Xi_{1}$ in (6) is the Fujikawa measure for these integrals. The role of $\Xi_{1}$ is as follows.

$(\alpha)$ It has to render the path integral measure invariant under changes in the normalized gauge parameter, e.g., $\hat{t}_{1}$, which runs from 0 to 1 . This can be done by choosing the factor $\Xi\left(t_{1}\right)$ such that the product $\int_{0}^{1} d \hat{t}_{1} J \Xi_{1}$ is independent of $\hat{t}_{1}$. Alternatively, this can be achieved by choosing a value of $\hat{t}_{1}$ where the Jacobian determinant of the integral is independent of changes in $\hat{t}_{1}$.

$(\beta)$ The above procedure $(\alpha)$ leaves a freedom in terms of a multiplicative factor of the action value $S^{b}$. It is imposed that $\Xi$ has to multiply the measure with the necessary power of $S^{b}$ in order to keep the integrand nonzero and finite for $S \rightarrow 0$.

$(\gamma)$ The above procedures $(\alpha)$ and $(\beta)$ leave a freedom in terms of a multiplicative factor containing the external constants $m$ and $t_{f}$. Without loss of generality, $m$ can be chosen to be one, and the external power of $t_{f}^{c}$ is, like always, determined from an additional normalization condition.

As described above, a crucial step of the recipe for the calculation of path integrals with the hidden local symmetry is to rewrite the integral in terms of the action as an integration variable. For the TPCF (6), this can be achieved by a coordinate change $\left\{x_{1} \rightarrow S, t_{1} \rightarrow \hat{t}_{1}\right\}$ with

$$
x_{1}=\frac{\sqrt{\left(t_{f}^{2}-(S / m)^{2}\right)\left((S / m)^{2}-\left(t_{f}-2 t_{1}\right)^{2}\right)}}{2(S / m)},
$$

$$
t_{1}=\frac{(S / m)^{2}}{2 t_{f}} \hat{t}_{1},
$$

where the integration goes for $S:-t_{f} m \ldots 0$ and $\hat{t}_{1}: 0 \ldots 1$. However, for convenience, the minus sign of the action $S$ can be absorbed in the redefinition of the integration variable $\tilde{S}=-S$ with the corresponding change of the boundary $-t_{f} m \rightarrow t_{f} m$. The Jacobian determinant for the applied transformations is

$$
\begin{aligned}
J= & \frac{1}{\sqrt{\left(m t_{f}\right)^{2}-\tilde{S}^{2}}} \\
& \cdot \frac{\tilde{S}^{4}\left(\hat{t}_{1}^{2}-1\right)-2 m^{2} \tilde{S}^{2} \hat{t}_{1} t_{f}^{2}+m^{4} t_{f}^{4}}{4 m^{3} t_{f} \sqrt{\tilde{S}^{2}-\left(\left(t_{f} m\right)-\tilde{S}^{2} \hat{t}_{1} /\left(m t_{f}\right)\right)^{2}}} .
\end{aligned}
$$

Thus,

$$
K^{(1)}=\mathcal{N} \cdot \int_{0}^{t_{f} m} d \tilde{S} \int_{0}^{1} d \hat{t}_{1} J \Xi_{1} e^{-i \tilde{S}}
$$

Since from condition $(\alpha)$ one demands

$$
\frac{d}{d \hat{t}_{1}} J \cdot \Xi_{1}=0
$$

one can simply choose the Fujikawa determinant as the inverse of the $\hat{t}_{1}$-dependent second term in Eq. (9):

$$
\Xi_{1}=\frac{4 m^{3} t_{f} \sqrt{\tilde{S}^{2}-\left(\left(t_{f} m\right)-\tilde{S}^{2} \hat{t}_{1} /\left(m t_{f}\right)\right)^{2}}}{\tilde{S}^{4}\left(\hat{t}_{1}^{2}-1\right)-2 m^{2} \tilde{S}^{2} \hat{t}_{1} t_{f}^{2}+m^{4} t_{f}^{4}} .
$$

Equivalently, one can also apply the principle of minimal sensitivity (PMS) [27-30] by choosing the optimal (symmetric and stable) value $\hat{t}_{1}^{\text {opt }}=\frac{m^{2} t_{f}^{2}}{\tilde{S}^{2}}$, as outlined in $(\alpha)$. This alternative approach will be used in higher spatial dimensions, as shown in Sec. II G.

A subsequent normalization with powers of $\tilde{S}$ and $t_{f}$ as mentioned in $(\beta)$ and $(\gamma)$ gives the TPCF

$$
\begin{aligned}
K_{O}^{(1)}\left(0, t_{f}\right)= & \mathcal{N} \int_{0}^{t_{f} m} d \tilde{S} \frac{m}{\sqrt{\left(m t_{f}\right)^{2}-(\tilde{S})^{2}}} \\
& \times \exp (-i \tilde{S})\left(\int_{0}^{1} d \hat{t}_{1}\right) \\
= & \mathcal{N} \frac{m \pi}{2}\left(J_{0}\left(t_{f} m\right)-i H_{0}\left(t_{f} m\right)\right),
\end{aligned}
$$

where $J_{0}$ is the Bessel function of the first kind and $H_{0}$ is the Struve function. This result can be stated in an explicitly relativistic invariant form, by noticing that

$$
\begin{gathered}
m t_{f}=m \sqrt{\left(x_{f}-x_{i}\right)^{\mu}\left(x_{f}-x_{i}\right)_{\mu}} \equiv m\left|x_{f}-x_{i}\right|, \text { such that } \\
K_{O}^{(1)}\left(x_{i}^{\mu}, x_{f}^{\mu}\right)= \\
-i \frac{m \pi}{2}\left(J_{0}\left(m\left|x_{f}-x_{i}\right|\right)\right. \\
\left.-i H_{0}\left(m\left|x_{f}-x_{i}\right|\right)\right) .
\end{gathered}
$$

The subindex " $O$ " refers to timelike orthochronous, since for this TPCF all virtual paths were timelike and respected the time ordering of $t_{f}>t_{1}>0$.

We notice that both functions $J_{0}(z)$ and $H_{0}(z)$ are independent solutions of the Bessel differential equation. 
Therefore, as proved in the Appendix A, when $z=m|x|$, they are also independent solutions of the KleinGordon equation. One can, thus, conclude that the TPCF is itself a solution of the Klein-Gordon equation for $|x|>0$, i.e.,

$$
\left(\square_{x}+m^{2}\right) K_{O}^{(1)}(m|x|)=0 .
$$

In a later section, the relation between this TPCF and the Feynman propagator of a scalar field will be discussed in more detail.

\section{The $N+1$-step TPCF}

The full TPCF is defined as the sum of all possible TPCFs $K_{O}^{(n)}$ with $n$ intermediate steps:

$$
K_{O}=\left.\sum_{n=1}^{\infty} K_{O}^{(n)}\right|_{\mathrm{NOC}}
$$

Since $K_{O}^{(1)}$ was calculated in the previous section, let us now continue with $K_{O}^{(2)}$, by showing that the contribution of this TPCF is zero due to the "no overcounting" condition $\left.\right|_{\text {NOC }}$.

In this proof, a "spatial flip" symmetry will be assumed. The $K_{O}^{(2)}$ TPCF can be constructed by the following steps:

(i) First, choose an arbitrary intermediate point $x_{1}^{\mu}$. This situation is shown in Fig. 3. This point corresponds to an action value $S_{1}=S_{c l}\left(0, x_{1}^{\mu}\right)+S_{c l}\left(x_{1}^{\mu}, x_{f}^{\mu}\right)$.

However, due to the symmetry (b) from all the points with the same action value, indicated by the blue contour in the first panel in Fig. 3, only one has to be counted. As indicated in this panel, we will choose the point $x_{1}^{\prime \mu}$ which is lightlike with the initial position 0 .

(ii) Having fixed the point $x_{1}^{\prime \mu}$, one chooses an arbitrary second point $x_{2}^{\mu}$, which is in the future light cone of $x_{1}^{\prime \mu}$ and in the past light cone of $x_{f}^{\mu}$. This situation is shown in the second panel in Fig. 3. This choice corresponds to a different action; while now keeping $x_{1}^{\prime \prime}$ fixed, again due to symmetry (b), the point $x_{2}^{\mu}$ can be replaced by $x_{2}^{\prime \mu}$ which is lightlike with the initial position 0 .

(iii) Now one observes that the first two steps of the path $x_{i}^{\mu} \rightarrow x_{1}^{\prime \mu} \rightarrow x_{2}^{\prime \mu} \rightarrow x_{f}^{\mu}$ are on a straight light curve. This path is already considered in the construction of the $K_{O}^{(1)} \mathrm{TPCF}$ and, thus, due to the NOC condition arising from Weyl invariance (c), it is not to be considered again. This happens for all the paths one attempts to add for the $K_{O}^{(2)}$ construction.

(iv) The stepwise construction outlined above for $K_{O}^{(2)}$ can by iteration be generalized to an arbitrary
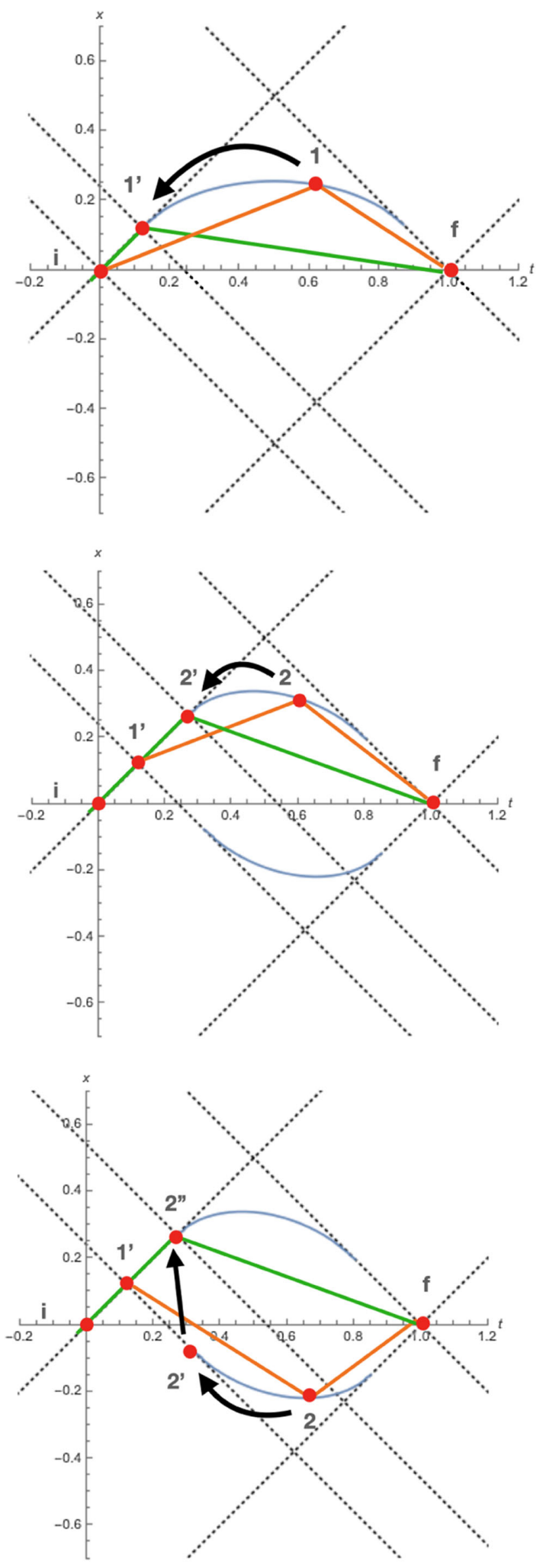

FIG. 3. The graphical sequence of steps (see the main text) leading to the construction of the orthochronous TPCF.

number of timelike orthochronous steps: $K_{O}^{(n)}$ brings nothing new with respect to $K_{O}^{(1)}$ and, thus, by virtue of the NOC condition, $K_{O}^{(n)}$ has to be excluded. 
One concludes that the full orthochronous TPCF is given by the TPCF with one intermediate integration:

$$
\begin{aligned}
K_{O}=K_{O}^{(1)}= & \mathcal{N} \frac{m \pi}{2}\left(J_{0}\left(m\left|x_{f}-x_{i}\right|\right)\right. \\
& \left.-i H_{0}\left(m\left|x_{f}-x_{i}\right|\right)\right) .
\end{aligned}
$$

There is one implicit assumption in this construction in $1+1$ dimensions, which is shown in Fig. 3. If the second integration point $x_{2}^{\mu}$ happens to be below the straight line connecting $x_{1}^{\prime \mu}$ and $x_{f}^{\mu}$, the continuous transformation of the point $x_{2}^{\mu}$ to a light cone of the point $x_{1}^{\prime \mu}$ brings it to the point $x_{2}^{\prime \mu}$. However, the light lines $x_{1}^{\prime \mu} \rightarrow x_{2}^{\prime \mu}$ and $x_{i}^{\mu} \rightarrow x_{1}^{\prime \mu}$ are going into opposite spatial directions. In order to get the points aligned, one needs to spatially flip from $x_{2}^{\prime \mu} \Rightarrow x_{2}^{\prime \prime \mu}$, as indicated on the right-hand side of Fig. 3. The assumption one has to make here is that this discrete flip forms part of the hidden (local) symmetry of equivalent paths (b). This is an isolated problem of $1+1$ dimensions, since, in spatial dimensions higher than one, (b) contains also spatial rotations, which allow for a continuous transformation from $x_{2}^{\prime \mu}$ to $x_{2}^{\prime \prime \mu}$. Thus, from a higher-dimensional perspective, the discrete spatial flip can be understood as a continuous rotation in the higher-dimensional theory. Nevertheless, it is interesting to explore the possibility that this flip is excluded from the symmetry (b) in $1+1$ dimensions. This will be done below.

\section{Spacelike, timelike, orthochronous TPCFs and the Feynman propagator}

The orthochronous TPCF $K_{O}$ (13) is not the familiar position space Feynman propagator of a scalar field in $1+1$ dimensions:

$$
\begin{aligned}
K_{F}\left(0, t_{f}\right) & =\mathcal{N} \int_{1}^{\infty} d y \frac{m}{\sqrt{y^{2}-1}} e^{-i t_{f} m y} \\
& =-\mathcal{N} \frac{i \pi}{2} H_{0}^{(2)}\left(\left|t_{f}\right| m\right),
\end{aligned}
$$

where $H_{0}^{(2)}$ is the Hankel function. Note that Eq. (18) corresponds to the well-known form of the TPCF in momentum space:

$$
K_{F}(k)=\frac{-i}{k^{2}-m^{2}+i \epsilon} .
$$

To understand the physical meaning of this difference and how the TPCFs $K_{O}$ and $K_{F}$ are connected, it is helpful to consider the additional space-time regions $V$ for the intermediate steps of the TPCF (6). This is done on the top subfigure of Fig. 4. One can distinguish between three different pure cases.

(I) The virtual paths are timelike orthochronous, which means that they are in the future light cone of the
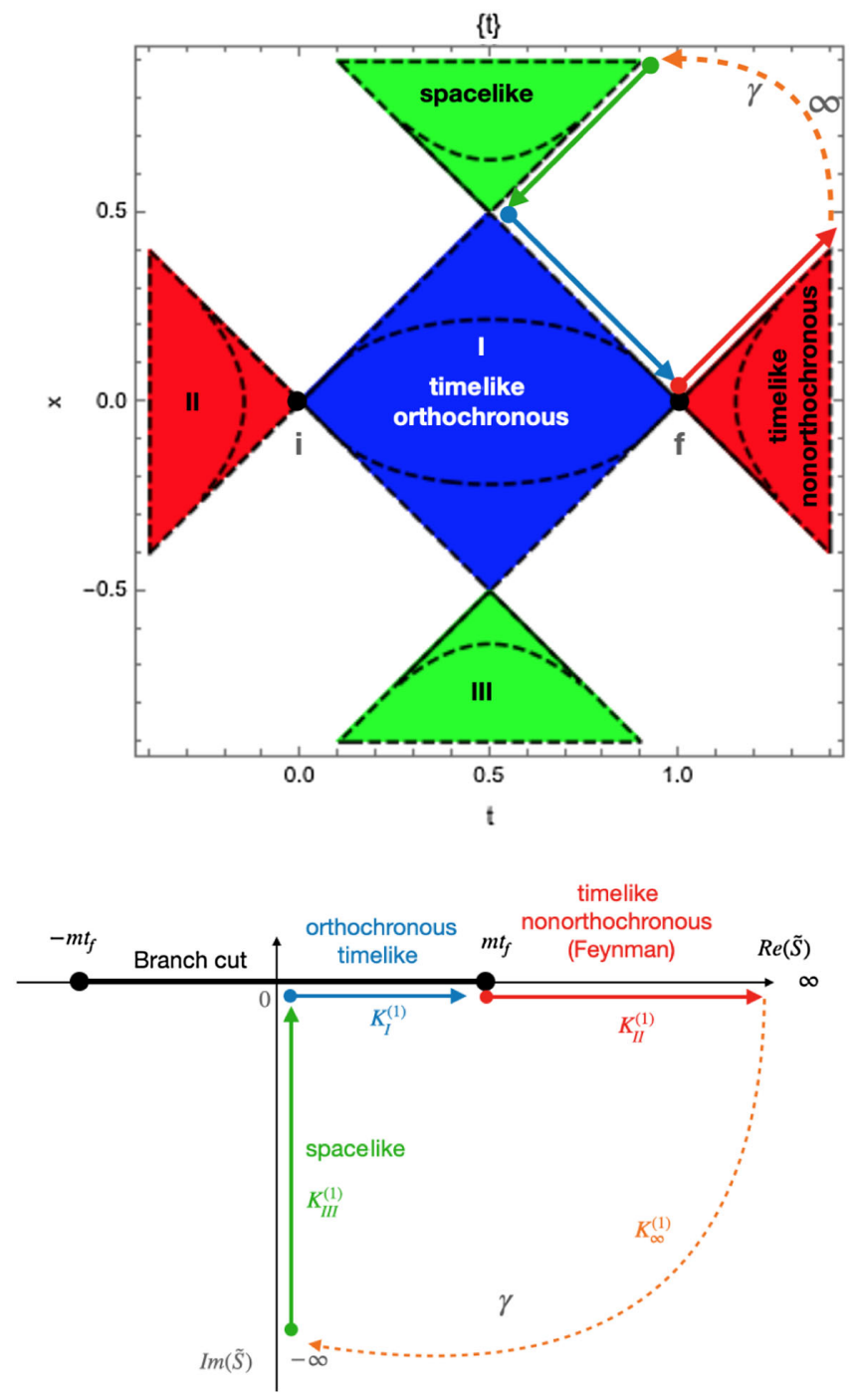

FIG. 4. Top: topology of paths with one intermediate step. Blue stands for timelike orthochronous, red for timelike nonorthochronous, and green for spacelike. The arrows indicate the integration contour shown in the right panel. Bottom: sections and integration contour in the complex $\tilde{S}$ plane.

initial position and the past light cone of the final position. These paths are indicated by the blue region of Fig. 4. They lead to the TPCF $K_{I}^{(1)}=$ $K_{O}^{(1)}$ given in Eq. (13).

(II) The virtual paths are timelike but not orthochronous, which means that the "intermediate" step lies either in the future light cone of both 0 and $t_{f}$ or in the past light cone of both 0 and $t_{f}$. These paths are indicated by the red regions in Fig. 4. Repeating the calculation from Sec. II B, one finds that the integral over this type of paths gives the Feynman propagator of a scalar field:

$$
K_{I I}^{(1)}=K_{F}
$$


given in Eq. (18), which can be seen by the change of integration variables $y=\tilde{S} /\left(t_{f} m\right)$.

(III) The third possibility is that all virtual paths are spacelike, which is indicated by the green regions in Fig. 4. These paths have imaginary action, and by using the methods of Sec. II B one finds the TPCF for spacelike virtual paths:

$$
\begin{aligned}
K_{S}^{(1)} & \equiv K_{I I I}^{(1)}=-\mathcal{N} \int_{0}^{\infty} d y \frac{e^{-t_{f} m y}}{\sqrt{y^{2}+1}} \\
& =\mathcal{N} \frac{\pi}{2}\left(Y_{0}\left(t_{f} m\right)-H_{0}\left(t_{f} m\right)\right)
\end{aligned}
$$

where $Y_{0}$ is the Bessel function of the second kind and $H_{0}$ is the Struve function.

There are further regions where the action contains both a real and an imaginary part. These regions, which are left blank in the top subfigure in Fig. 4, would correspond to paths which cross their light cones along the way. As shown in Appendix B, such mixed paths cannot be tackled with methods proposed in this paper. They will not be considered in the construction of the TPCF. This means that all paths are "free to do what they want," as long as they stay within their respective causal pattern. Note that one can perform a very similar path integral construction for paths which mix different causal regions, if one considers in the action (4) absolute values instead of square root contributions for each step. This will be left to a future study.

For the regions considered here, one realizes that the three TPCFs $K_{I}^{(1)}, K_{I I}^{(1)}$, and $K_{I I I}^{(1)}$ all arise from integrating the function

$$
f\left(y, t_{f} m\right)=\frac{1}{\sqrt{y^{2}-1}} e^{-i y t_{f} m}
$$

along different sections of the complex plane. The integration variable is given from the action by $y=\tilde{S} /\left(m t_{f}\right)$. Because of the square root in the denominator, this function possesses a branch cut along the real axis, as depicted in Fig. 4. Thus, as shown in the bottom subfigure in Fig. 4, the integrals over (22) can be plugged together to form a closed contour $\gamma=\gamma(y)$. Since this contour neither crosses the branch cut nor contains zeros or poles, one can apply the residue theorem

$$
\oint_{\gamma} d y f\left(y, t_{f} m\right)=K_{I}^{(1)}+K_{I I}^{(1)}+K_{\infty}^{(1)}+K_{I I I}^{(1)}=0 .
$$

Since, due to the exponential factor, the angular integral at infinity vanishes $K_{\infty}^{(1)}=0$, one finds that the Feynman propagator can be obtained either by an integration over all timelike nonorthochronous paths (20) or by an integration over all timelike orthochronous paths plus all spacelike paths:

$$
K_{F}\left(t_{f} m\right)=-\left(K_{S}^{(1)}\left(t_{f} m\right)+K_{O}^{(1)}\left(t_{f} m\right)\right) .
$$

The generalization of the results (20) and (24) for the $n$-step TPCF is straightforward, and it follows from the same arguments already presented in Sec. IIC, if one considers the following adjustments.

(i) One connects virtual paths of the same type: e.g., orthochronous with orthochronous, spacelike with spacelike, or nonorthochronous with nonorthochronous.

(ii) The discrete symmetry for spacelike paths is still a spatial "flip," while the discrete symmetry for nonorthochronous timelike paths is a flip from the pastpast to the future-future light cone or vice versa. In summary, one finds

$$
\begin{gathered}
K_{I}\left(t_{f} m\right)=K_{I}^{(1)}\left(t_{f} m\right)=K_{O}\left(t_{f} m\right), \\
K_{I I}\left(t_{f} m\right)=K_{I I}^{(1)}\left(t_{f} m\right)=K_{F}\left(t_{f} m\right), \\
K_{I I I}\left(t_{f} m\right)=K_{I I I}^{(1)}\left(t_{f} m\right)=K_{S}\left(t_{f} m\right),
\end{gathered}
$$

and

$$
K_{F}\left(t_{f} m\right)=-\left(K_{S}\left(t_{f} m\right)+K_{O}\left(t_{f} m\right)\right) .
$$

In light of the above analytic relation, it is interesting to convince oneself geometrically that if one combines an orthochronous step with a spatial step some paths can be transformed by the use of (b) to a nonorthochronous path appearing in $K_{I I}^{(1)}$.

\section{E. Comparison to the TPCFs of the Klein-Gordon field}

The most common TPCFs for scalar fields are the advanced $\Delta_{+}$, retarded $\Delta_{-}$, causal $\Delta_{C}$, Wightman $\Delta_{H}$, and the Feynman propagator $K_{F}$. An important distinction between all these TPCFs is their behavior under sign changes of their argument $t_{f} \leftrightarrow-t_{f}$.

The relativistic point particle action (4) and its path integrals are blind to such changes $t_{f} \leftrightarrow-t_{f}$, and any distinction using this criterion would not be related to the causal structure of the action discussed in the previous subsection. Thus, we are working under the assumption of positive $t_{f}>0$ (or even transformation behavior), but the resulting TPCF $K_{O}$ contains both even and odd functions of $t_{f}$.

From the above-mentioned TPCFs for the Klein-Gordon equation, only the Feynman propagator has the desired even transformation behavior $K_{F}\left(t_{f}\right)=K_{F}\left(-t_{f}\right)$. Thus, only this TPCF appears in the comparison to the results for the relativistic point particle calculated here. Instead, for example, the causal TPCF $\Delta_{C}=\Delta_{+}-\Delta_{-}$is not directly related to the timelike orthochronous TPCF $K_{O}$. 


\section{F. Without spatial flip symmetry and Feynman's checkerboard}

In this subsection, part of the procedure from the previous subsections will be repeated, but the discrete flips in $1+1$ dimensions will be taken to be as physically inequivalent paths and not part of the local symmetry (b). The orthochronous TPCF arising from this will be called $K_{O C}^{(l)}$ as opposed to the $K_{O}^{(l)}$ TPCF from the previous section.

The construction goes as follows.

(i) For the TPCF $K_{O C}^{(1)}$, one chooses the first point $x_{1}^{\mu}$ and uses the symmetry (b) to shift to a physically equivalent point $x_{1}^{\mu} \Rightarrow x_{1}^{\prime \mu}$. Already, here one can distinguish between $K_{O}^{(1)}$ going up and $K_{O}^{(1)}$ going down, which have to be summed. This is shown in the top panel in Fig. 5.

(ii) For the TPCF $K_{O C}^{(2)}$, one chooses the second point $x_{2}^{\mu}$ while taking $x_{1}^{\prime \mu}$ as given and uses the symmetry (b) to shift to a physically equivalent point $x_{2}^{\mu} \Rightarrow x_{2}^{\prime \mu}$, which is lightlike with $x_{1}^{\prime \prime}$. If this point is the straight continuation of the light line $x_{i}^{\mu} \rightarrow x_{1}^{\prime \mu}$, the discussion from the previous section applies and this contribution is discarded. If, to the contrary, light lines $x_{i}^{\mu} \rightarrow x_{1}^{\prime \mu}$ and $x_{1}^{\prime \mu} \rightarrow x_{2}^{\prime \mu}$ are going in opposite spatial directions, as shown in the middle panel in Fig. 5, this path is a new contribution which forms part of $K_{O C}^{(2)}$.

(iii) For the TPCF $K_{O C}^{(l)}$, the construction continues analogously. Countable new paths occur only when there is a kink between two subsequent light lines. For $K_{O C}^{(5)}$, this is shown in the bottom panel in Fig. 5.

One realizes that, for each step from $K_{O C}^{(l)}$ to $K_{O C}^{(l+1)}$, the available maximal absolute value of the action $\tilde{S}_{(l+1)}$ reduces, such that for $\lim _{l \rightarrow \infty} K_{O C}^{(l)}$ the entire path is lightlike.

The total TPCF is now

$$
K_{O C}\left(x_{i}^{\mu}, x_{f}^{\mu}\right)=\sum_{l=1}^{\infty} K_{O C}^{(l)}\left(x_{i}^{\mu}, x_{f}^{\mu}\right)
$$

which is harder to work out than the TPCF (17). First, as in Sec. II B, one can write out the integral expression for each of the TPCFs $K_{O C}^{(l)}$ in terms of the absolute value of the corresponding action:

$$
\begin{aligned}
& K_{O C}\left(x_{i}^{\mu}, x_{f}^{\mu}\right) \\
& =\lim _{N \rightarrow \infty} \sum_{l=1}^{N} \omega^{l} \prod_{j=1}^{l}\left(\int_{0}^{\tilde{S}_{j-1}} d S_{j} \frac{1}{\sqrt{\tilde{S}_{j-1}^{2}-\tilde{S}_{j}^{2}}}\right) e^{-i\left(\tilde{S}_{l}\right)},
\end{aligned}
$$

where $\tilde{S}_{0}=m \sqrt{\left(x_{i}-x_{f}\right)^{\mu}\left(x_{i}-x_{f}\right)_{\mu}}$ and $\omega$ is the measure weight associated to each change in direction, where a
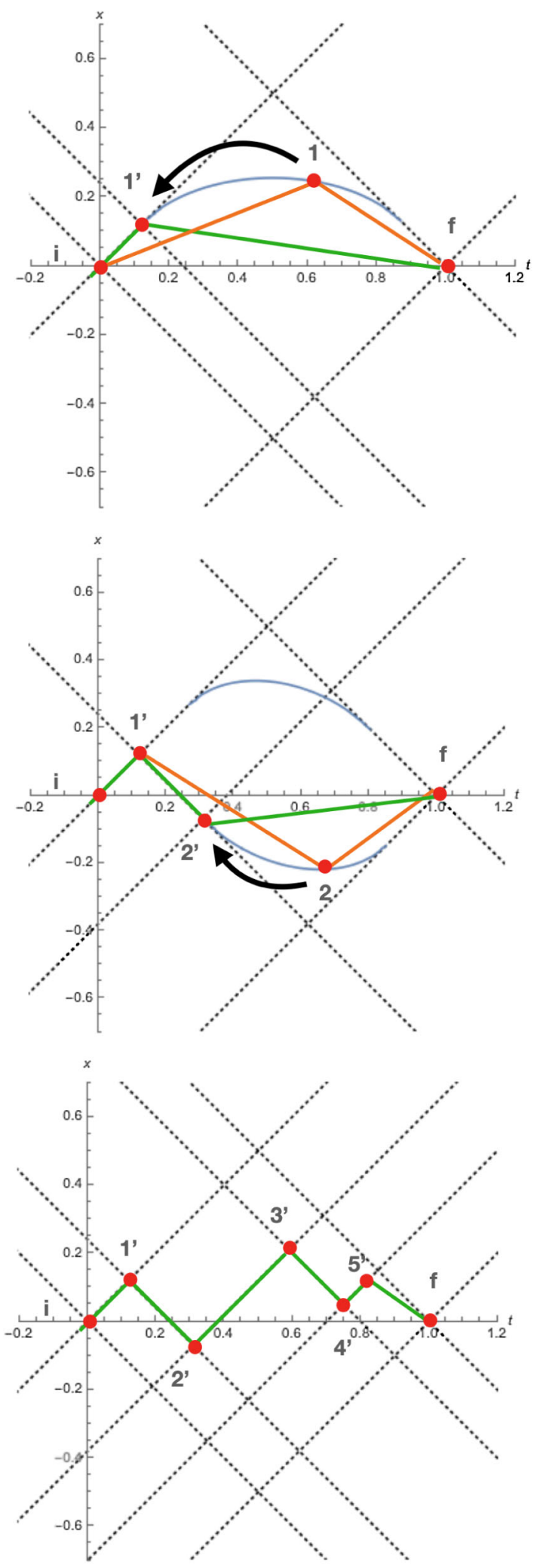

FIG. 5. The topological construction of the paths leading to the orthochronous TPCF is displayed.

discrete realization of (b) was avoided. This is a sum over all lightlike paths with $l$ turns, where only one of the steps is timelike. In the right panel in Fig. 5, this step was chosen as 
the last one $x_{5}^{\prime} \rightarrow x_{f}$. By approaching the construction from the left and from the right, the nonlightlike step could have been any pair $x_{j-1}^{\prime \mu} \rightarrow x_{j}^{\prime \prime}$. In any case, for large $l \rightarrow \infty$, the typical value for the remaining action $\sim \sqrt{\left(x_{j-1}^{\prime}-x_{j}^{\prime}\right)^{\mu}\left(x_{j-1}^{\prime}-x_{j}^{\prime}\right)_{\mu}}$ would approach zero $\tilde{S}_{l} \rightarrow 0$.

Thus, for a large number of steps, the TPCF $K_{O C}^{(l)}$ contains a discrete sum over all lightlike paths with the additional measure weight $\omega^{l}$ :

$$
K_{O C}\left(x_{i}^{\mu}, x_{f}^{\mu}\right)=\lim _{N \rightarrow \infty} \sum_{l=1}^{N} \omega^{l} \Phi(l, N),
$$

where $\Phi(l, N)$ is the number of paths with $l$ turns for a given discretization $N$.

A beautiful realization of this concept is given in terms of the Feynman checkerboard, where the weight is chosen to be [31]

$$
\omega=i \frac{m c^{2}}{\hbar} \frac{\left(t_{f}-t_{i}\right)}{N}
$$

Conceptually, this choice for the weight factor arises as a consequence of Heisenberg's uncertainty principle, $\Delta x \Delta p \geq \hbar$. For a massive relativistic particle, the minimum uncertainty in momentum is $\Delta p \sim m c$, and, therefore, the leaps that the particle experiences should satisfy $\Delta x \geq \hbar /(m c)$; i.e., they cannot be smaller than the Compton wavelength $\bar{\lambda}_{C}$. For a finite path consisting on a large number of such "leaps" with $\Delta x \simeq c \Delta t$, the ratio $\omega=i \Delta x / \bar{\lambda}_{C}=i m c^{2} \Delta t / \hbar$ thus provides the appropriate weight such that the path integral is dominated by those trajectories where the uncertainty principle inequality is satisfied in the majority of steps.

In the checkerboard, $\Phi(l, N)$ is approximated by a lattice of $N$ lightlike steps with $l$ turns of the spatial direction. Furthermore, a distinction between the direction $(+,-)$ of the first and last steps is made. With this distinction, the TPCF takes the form of a $2 \times 2$ matrix:

$K_{O C}\left(x_{i}^{\mu}, x_{f}^{\mu}\right)=\lim _{N \rightarrow \infty} \sum_{l=1}^{N} \omega^{l}\left(\begin{array}{ll}\Phi_{++}(l, N) & \Phi_{+-}(l, N) \\ \Phi_{-+}(l, N) & \Phi_{--}(l, N)\end{array}\right)$,

where, for example, $\Phi_{++}(l, N)$ stands for the number of paths that leave moving right and arrive moving right. Interestingly, Eq. (33) turns out to be identical to the Dirac propagator in position space $1+1$ dimensions. As discussed in detail in Ref. [31], the problem of calculating the coefficients $\Phi_{\alpha \beta}(l, N)$ can be cast in the form of an Ising model, defining the instantaneous direction of the $j$ th step by a two-valued spin $\sigma_{j}= \pm$, such that the net number of steps in the positive $x$ direction is

$$
M=\sum_{j=1}^{N} \sigma_{j}
$$

whereas the total number of flips $l$ corresponds in this language to

$$
l=\frac{1}{2} \sum_{j=1}^{N-1}\left(1-\sigma_{j} \sigma_{j+1}\right)=\frac{N-1}{2}-\frac{1}{2} \sum_{i=1}^{N-1} \sigma_{j} \sigma_{j+1} .
$$

Therefore, after introducing an integral representation for the Kronecker delta,

$$
\delta_{M, \sum_{j} \sigma_{j}}=\int_{-\pi}^{\pi} \frac{d \theta}{2 \pi} e^{i \theta\left(\sum_{j=1}^{N} \sigma_{j}-M\right)},
$$

the elements of the TPCF Eq. (33) correspond to the sum (for $\alpha= \pm$ and $\beta= \pm$ not contracted, but fixed and in correspondence with $\Phi_{\alpha \beta}$ )

$$
\begin{aligned}
\sum_{l=1}^{N} \Phi_{\alpha \beta}(l, N) \omega^{l} & =\sum_{\sigma_{2}= \pm} \cdots \sum_{\sigma_{N-1}= \pm} \omega^{l} \\
& =\int_{-\pi}^{\pi} \frac{d \theta}{2 \pi} e^{i M \theta} e^{-\frac{i}{2} \theta(\alpha+\beta)}\left[\hat{T}^{N-1}\right]_{\alpha \beta}
\end{aligned}
$$

where, by analogy with the Ising model, the transfer matrix is defined by

$$
\hat{T}_{\sigma, \sigma^{\prime}}=\exp \left(\nu \sigma \sigma^{\prime}+i \frac{\theta}{2}\left(\sigma+\sigma^{\prime}\right)-\nu\right)
$$

and $\nu=-\frac{1}{2} \log (\omega)$. Applying a similarity transformation that diagonalizes the transfer matrix, a saddle point calculation is performed to determine the stationary value of the parameter $\sin \theta=i \mu \omega v / \sqrt{1-v^{2}}$, with $\mu= \pm 1$ and $v=$ $\left(x_{f}-x_{i}\right) /(-i N \omega)$ the linear "speed" of the particle (in natural units such that $c=\hbar=1$ ). Therefore, the corresponding expression obtained for the propagator from this procedure is the famous checkerboard result [31]

$$
\begin{aligned}
\sum_{l=1}^{N} \Phi_{\alpha \beta}(l, N) \omega^{l}= & \omega \sum_{\mu= \pm} \int \frac{d p}{2 \pi} \frac{1}{2}\left[1+\mu \frac{m \sigma_{x}-p \sigma_{z}}{\sqrt{p^{2}+m^{2}}}\right]_{\alpha \beta} \\
& \times e^{i p\left(x_{f}-x_{i}\right)} e^{i \mu \sqrt{p^{2}+m^{2}}} \\
= & \frac{\omega}{2 \pi} \int d p e^{i p\left(x_{f}-x_{i}\right)}\left[e^{i\left(m \sigma_{x}-p \sigma_{z}\right)}\right]_{\alpha \beta} .
\end{aligned}
$$

\section{G. Higher-dimensional generalization}

The higher-dimensional generalization of calculation of the $(1+1)$-dimensional TPCF can be done straightforwardly. To vary the construction, the PMS will be applied. In $(1+d)$ dimensions, the TPCF (6) reads 


$$
K^{(1)}=\mathcal{N} \cdot \int d t_{1} \int_{V} d^{d} x_{1} \Xi_{1} e^{i S_{1}}
$$

First, one can write the $d$ spatial integrals in spherical coordinates:

$$
K^{(1)}=\mathcal{N} \cdot \int d t_{1} \int_{V} d\left|x_{1}\right| d \Omega_{d-1}\left|x_{1}\right|^{d-1} \Xi_{1} e^{i S_{1}}
$$

The integrand is independent of the angular coordinates, and, thus, the integral over $d \Omega_{d-1}$ gives $2 \pi^{(d-1) / 2} /$ $\Gamma((d-1) / 2)$, which is the surface of the unit sphere in $d-1$ dimensions. This constant factor can be absorbed into the normalization constant. Now one proceeds with a change in the integration variables from $\left|x_{1}\right| \rightarrow S$ given in Eq. (7), keeping the $t_{1}$ integration untouched. The integral now reads

$K^{(1)}=\mathcal{N} \cdot \int_{-t_{f} m}^{0} d S \int_{t_{f} / 2-S^{2} /\left(2 m^{2} t_{f}\right)}^{t_{f} / 2+S^{2} /\left(2 m^{2} t_{f}\right)} d t_{1} J \cdot\left|x_{1}\right|^{d-1} \Xi_{1} e^{i S}$.

After a change of variables $S=-\tilde{S}$, we obtain

$K^{(1)}=\mathcal{N} \cdot \int_{0}^{t_{f} m} d \tilde{S} \int_{t_{f} / 2-\tilde{S}^{2} /\left(2 m^{2} t_{f}\right)}^{t_{f} / 2+\tilde{S}^{2} /\left(2 m^{2} t_{f}\right)} d t_{1} J \cdot\left|x_{1}\right|^{d-1} \Xi_{1} e^{-i \tilde{S}}$

The factor $J \cdot\left|x_{1}\right|^{d-1}$ is a function of $\tilde{S}$, where $\left|x_{1}\right|$ is given by Eq. (7) and

$$
J=\frac{m^{4} t_{f}^{2}\left(t_{f}-2 t_{1}\right)^{2}-\tilde{S}^{4}}{2 m \tilde{S}^{2} \sqrt{\left(m^{2} t_{f}^{2}-\tilde{S}^{2}\right)\left(\tilde{S}^{2}-m^{2}\left(t_{f}-2 t_{1}\right)^{2}\right)}}
$$

Now one has to get rid of the $t_{1}$ dependence. Instead of imposing a $t_{1}$-dependent Fujikawa factor $\Xi_{1}=\Xi_{1}\left(t_{1}\right)$, we will now choose an optimal $t_{1}$ such that

$$
\left.\frac{d}{d t_{1}} J \cdot\left|x_{1}\right|^{d-1}\right|_{t_{1}=t_{1, \mathrm{opt}}}=0
$$

The solutions to this condition are

$$
t_{1, \mathrm{opt}}=\left\{\begin{array}{l}
\frac{t_{f}}{2} \\
\frac{m t_{f} \pm \tilde{S}}{2 m} \\
\frac{t_{f}}{2} \pm \frac{m^{4} \tilde{S}^{2} t_{f}^{2}\left(2 m^{2} t_{f}^{2}+\tilde{S}^{2} d\right) d}{2 m^{4} t_{f}^{2} d}
\end{array}\right.
$$

One realizes that the only solution which lies in the allowed integration range of $d t_{1}$ is

$$
t_{1, \mathrm{opt}}=\frac{t_{f}}{2}
$$

With this, the TPCF reads, up to a normalization constant,

$$
K^{(1)}=\mathcal{N} \cdot \int_{0}^{t_{f} m} d \tilde{S}\left(m^{2} t_{f}^{2}-\tilde{S}^{2}\right)^{(d-2) / 2} \tilde{S} \Xi_{1} e^{-i \tilde{S}}
$$

According to the condition $(\alpha)$, this has to be finite for $\tilde{S} \rightarrow 0$ and with the right dimensions, which gives

$$
\Xi_{1}=\frac{1}{\tilde{S}\left(t_{f} m\right)^{d-2}}
$$

and, thus,

$$
K^{(1)}=\mathcal{N} \cdot \int_{0}^{t_{f} m} d \tilde{S}\left(1-\frac{\tilde{S}^{2}}{m^{2} t_{f}^{2}}\right)^{(d-2) / 2} e^{-i \tilde{S}}
$$

which is the TPCF in $1+d$ dimensions. In order to bring this to a more familiar form, one can perform a number of operations. The coordinate transformation

$$
\tilde{S}=y m t_{f}
$$

gives

$$
K^{(1)}=\mathcal{N} \cdot \int_{0}^{1} d y{\sqrt{y^{2}-1}}^{(d-2)} e^{-i m t_{f} y},
$$

where a factor of $i^{d-2}$ was absorbed in the normalization. Now one defines

$$
y=\frac{\sqrt{\vec{k}^{2}+m^{2}}}{m}
$$

which leads to

$$
K^{(1)}=\mathcal{N} \cdot \int_{0}^{i m} d k \frac{k^{d-1} e^{-i t_{f} \sqrt{\vec{k}^{2}+m^{2}}}}{\sqrt{\vec{k}^{2}+m^{2}}}
$$

The $d k$ integral can be written in terms of a $d$-dimensional integral:

$$
K^{(1)}=\mathcal{N} \cdot \int_{0}^{i m} d^{d} k \frac{e^{-i\left|t_{f}\right| \sqrt{\vec{k}^{2}+m^{2}}}}{\sqrt{\vec{k}^{2}+m^{2}}}
$$

where the assumption of $t_{f}>0$ was made explicit by writing $\left|t_{f}\right|$ and where the upper limit is for the radial component of $d k$. This absolute value can then be written in terms of a $\theta$ function prescription, which, in turn, can be written as an integral over $k_{0}$, leaving 


$$
K^{(1)}=\mathcal{N} \cdot \int_{-\infty}^{\infty} d k_{0} \int_{0}^{i m} d^{d} k \frac{e^{-i k_{0} t_{f}}}{k^{2}-m^{2}+i \epsilon} .
$$

This is the familiar momentum space representation of the TPCF, but with a different contour of integration, as explained before. The analytical expression for this integral, up to action-independent constants absorbed in the normalization coefficient, is

$$
\begin{aligned}
K_{O}^{(1)}\left(0, t_{f}\right)= & \mathcal{N} \cdot\left(m t_{f}\right)^{\frac{1-d}{2}}\left[J_{\frac{d-1}{2}}\left(m t_{f}\right)\right. \\
& \left.-i H_{\frac{d-1}{2}}\left(m t_{f}\right)\right],
\end{aligned}
$$

where $J_{\frac{d-1}{2}}$ is the Bessel function of the first kind, $H_{\frac{d-1}{2}}$ is the Struve function, and we used again the "orthochronous" prescription, generalizing Eq. (13). By the same arguments presented in the previous section, the generalization of the Feynman propagator to $1+d$ dimensions is

$$
\begin{aligned}
K_{F}^{(1)}\left(0, t_{f}\right) & =\mathcal{N} \cdot \int_{1}^{\infty} d y{\sqrt{y^{2}-1}}^{(d-2)} e^{-i m t_{f} y} \\
& =\mathcal{N}\left(m t_{f}\right)^{\frac{1-d}{2}} H_{\frac{d-1}{2}}^{(1)}\left(m t_{f}\right)
\end{aligned}
$$

where $H_{\frac{d-1}{2}}^{(1)}$ is the Hankel function of the order of $(d-1) / 2$ and all action-independent constants have been absorbed into the overall normalization. Finally, the generalized form of the TPCF for space virtual paths is given by (see Appendix $\mathrm{C}$ for details)

$$
\begin{aligned}
K_{S}^{(1)}\left(0, t_{f}\right) & =-\mathcal{N} \cdot \int_{0}^{\infty} d y{\sqrt{y^{2}+1}}^{(d-2)} e^{-m t_{f} y} \\
& =\mathcal{N} \cdot\left(m t_{f}\right)^{\frac{1-d}{2}}\left[Y_{\frac{d-1}{2}}\left(m t_{f}\right)-H_{\frac{d-1}{2}}\left(m t_{f}\right)\right],
\end{aligned}
$$

where $Y_{\frac{d-1}{2}}$ is the Bessel function of the second kind. The proof for $N$ steps is completely analogous to the lowerdimensional case, so it will not be repeated.

\section{H. Higher-dimensional generalization of the checkerboard?}

The higher-dimensional generalization shown in the previous subsection works out so nicely that one is tempted to expect a similar result for the Feynman checkerboard, discussed in Sec. III. Certainly, the checkerboard approach has been explored in dimensions higher than $(1+1)$ [32-34]. Nevertheless, the elegance and interpretation in terms of a simple summation over lightlike paths is lost in these attempts. This unexpected fact can be easily understood in terms of the symmetry construction presented in this paper. As shown above, the checkerboard construction in $(1+1)$ dimensions arises naturally from counting discrete parity bounces as independent paths contributing to the TPCF. This was justified, since two such paths are not connected by a continuous symmetry transformation, and, thus, there is no overcounting in the sense of the conditions (a)-(c). However, in dimensions higher than $(1+1)$, any of these discrete bounces (see, e.g., Fig. 5) can be generated from a continuous rotation of the spatial directions. Such continuous transformations are overcountings in the sense of the conditions (a)-(c), and they have to be factored out. This is the reason why insisting on a checkerboard construction with more than one spatial dimension is unnatural.

\section{CONCLUSION}

In this paper, we studied the path-integral construction of the TPCFs of the free relativistic point particle. After analyzing the global and local symmetries of the action, we identified a local "hidden" symmetry that is usually disregarded in the literature. This symmetry corresponds to the invariance of the modulus of the 4-velocity with respect to Lorentz boosts and rotations. By taking explicit care of this symmetry and removing the associated redundant phase-space volume that leads to the overcounting of trajectories in the path integral by means of a Fujikawa prescription, we were able to obtain the correct results for the TPCFs that recover the Chapman-Kolmogorov property. Furthermore, we formulated the path integral explicitly in Minkowski space, by carefully taking into account the non-simply-connected structure of the Lorentz group. In a detailed topological analysis for the case of $D=1+1$ dimensions, we explicitly constructed different versions of the relativistic TPCF, for the different causal characteristics of the virtual paths. As a new result, we obtained a causal-orthochronous and a spacelike TPCF, and we also recovered the Feynman propagator. It was further shown that these three TPCFs are related due to a closed contour integral in the (complex-valued) Minkowski space. By means of this identity, the Feynman propagator could either be understood as the result of a PI over timelike but nonorthochronous paths, or it could be understood as PI over spacelike and timelikeorthochronous paths. The TPCFs in Secs. II B and II C were straightforwardly generalized to higher dimensions $D=d+1$.

Finally, based on the methods presented before, it has been shown how the Feynman checkerboard construction in $1+1$ dimensions arises naturally within this framework, and it has also been explained why a natural generalization of the checkerboard approach to higher dimensions does not work.

\section{ACKNOWLEDGMENTS}

E. M. was supported by Fondecyt (Fondo Nacional de Desarrollo Cientifico y Tecnologico, Chile) Grant No. 1190361 and by PIA (Programa de Investigacion Asociativa, ANID, Chile) Anillo Project No. ACT192023. 


\section{APPENDIX A: THE DIFFERENTIAL EQUATION SATISFIED BY THE TPCFS}

In this Appendix, we show that the different Bessel and related functions (such as Struve and Hankel) whose linear combinations give rise to the TPCFs obtained via path integration in this work, when their argument depends on the timelike interval $|x|=\sqrt{c^{2} t^{2}-\mathbf{x}^{2}}$, are themselves solutions of the Klein-Gordon equation for $|x|>0$.

In mathematical terms, we shall prove the following simple theorem.

Theorem.-If $y_{\alpha}(z)$ satisfies the Bessel equation

$$
z^{2} \frac{d^{2} y_{\alpha}}{d z^{2}}+z \frac{d y_{\alpha}}{d z}+\left(z^{2}-\alpha^{2}\right) y_{\alpha}(z)=0,
$$

then for $\alpha=0$ the function $y_{0}(z=m|x|)$ satisfies the Klein-Gordon equation

$$
\left(\square_{x}+m^{2}\right) y_{0}(m|x|)=0 .
$$

For simplicity, the proof will be given in spatial dimension $d=1$, where we have $|x|^{2}=c^{2} t^{2}-x^{2}$. Therefore, the following simple relations follow after application of the chain rule for partial derivatives:

$$
\begin{aligned}
\frac{1}{c} \frac{\partial|x|}{\partial t} & =\frac{c t}{|x|}, \\
\frac{1}{c^{2}} \frac{\partial^{2}|x|}{\partial t^{2}} & =\frac{1}{|x|}\left(1-\frac{c^{2} t^{2}}{|x|^{2}}\right)=-\frac{x^{2}}{|x|^{3}}
\end{aligned}
$$

and similarly

$$
\begin{aligned}
\frac{\partial|x|}{\partial x} & =-\frac{x}{|x|}, \\
\frac{\partial^{2}|x|}{\partial x^{2}} & =-\frac{1}{|x|}\left(1+\frac{x^{2}}{|x|^{2}}\right)=-\frac{c^{2} t^{2}}{|x|^{3}} .
\end{aligned}
$$

Let us now consider the action of the d'Alembert operator over a function $y_{0}(z=m|x|)$ that, by hypothesis, satisfies Eq. (A1). Therefore, after some straightforward algebra, we obtain

$$
\begin{aligned}
\square_{x} y_{0}(m|x|)= & \frac{1}{c^{2}} \frac{\partial^{2}}{\partial t^{2}} y_{0}(z)-\frac{\partial^{2}}{\partial x^{2}} y_{0}(z) \\
= & \frac{m}{c^{2}} \frac{\partial}{\partial t}\left(\frac{\partial|x|}{\partial t} \frac{d y_{0}(z)}{d z}\right)-m \frac{\partial}{\partial x}\left(\frac{\partial|x|}{\partial x} \frac{d y_{0}(z)}{d z}\right) \\
= & m\left(\frac{1}{c^{2}} \frac{\partial^{2}|x|}{\partial t^{2}}-\frac{\partial^{2}|x|}{\partial x^{2}}\right) \frac{d y_{0}}{d z} \\
& +m^{2}\left(\frac{1}{c^{2}}\left(\frac{\partial|x|}{\partial t}\right)^{2}-\left(\frac{\partial|x|}{\partial x}\right)^{2}\right) \frac{d^{2} y_{0}}{d z^{2}} .
\end{aligned}
$$

Substituting Eqs. (A3) and (A4) into Eq. (A5) and simplifying, we obtain

$$
\begin{aligned}
\square_{x} y_{0}(m|x|) & =m^{2} \frac{d^{2} y_{0}}{d z^{2}}+\frac{m}{|x|} \frac{d y_{0}}{d z} \\
& =m^{2}\left[\frac{d^{2} y_{0}}{d z^{2}}+\frac{1}{m|x|} \frac{d y_{0}}{d z}\right] \\
& =m^{2}\left[\frac{d^{2} y_{0}}{d z^{2}}+\frac{1}{z} \frac{d y_{0}}{d z}\right] \\
& =-m^{2} y_{0}(z),
\end{aligned}
$$

where in the last line we applied that, by hypothesis, the function $y_{0}(z)$ is a solution to the Bessel differential Eq. (A1) for $\alpha=0$. This proves the theorem, i.e., that $y_{0}(m|x|)$ is indeed a solution of the Klein-Gordon Eq. (A2) if it satisfies Eq. (A1).

Since all the TPCFs obtained in this work after the pathintegral procedures described in the text lead to linear combinations of Bessel and related functions (with $\alpha=0$ ), and since each of those functions satisfy the conditions of the theorem, then we conclude that each of the TPCFs constitute different solutions of the Klein-Gordon equation

$$
\left(\square_{x}+m^{2}\right) K(m|x|)=0 .
$$

\section{APPENDIX B: CONDITIONS ON THE WORLDLINES OF CONSTANT ACTION}

Intermediate points $\left(t_{1}, x_{1}\right)$ of the two-step construction that are equivalent in terms of the hidden symmetry (b) are shown as colored lines in Fig. 2 and as dashed lines in the upper part of Fig. 4. Along these lines, the action (4) is constant and contains two contributions:

$$
\begin{aligned}
S_{1} & =S_{01}+S_{1 f} \\
& =\sqrt{t_{1}^{2}-x_{1}^{2}}+\sqrt{\left(\left(t_{1}-t_{f}\right)-x_{1}\right)\left(\left(t_{1}-t_{f}\right)+x_{1}\right)},
\end{aligned}
$$

where we chose without loss of generality $t_{i}=x_{i}=x_{f}=0$. If both contributions $S_{01}$ and $S_{1 f}$ are real, the path is timelike (non)orthochronous, leading to $K_{I}^{(1)}\left(K_{I I}^{(1)}\right)$. If both contributions are imaginary, the path is spacelike, leading to $K_{I I I}^{(1)}$. In these sections (I, II, and III), the direction of the tangential vector $\left(\xi_{t}, \xi_{x}\right)$, generating the hiddengauge-equivalent lines, is obtained from the condition of constant action

$$
\xi_{t}\left(\partial_{t_{1}} S_{1}\right)+\xi_{x}\left(\partial_{x_{1}} S_{1}\right)=0 .
$$

One finds

$$
\frac{\xi_{t}}{\xi_{x}}=\frac{x_{1} S_{1}}{t_{1} S_{1 f}+S_{01}\left(t_{1}-f_{f}\right)} .
$$

There is also the possibility that one contribution (e.g., $S_{01}$ ) to a path is real and the other contribution is imaginary (e.g., $S_{1 f}$ ). These intermediate points are the blank regions in Fig. 4. For a hidden symmetry of the type (b) to exist, one 
needs a constant real and a constant imaginary part of the total action. Thus, there are two conditions of the type (B2), one for the real part and one for the imaginary part:

$$
\begin{aligned}
& \xi_{t}\left(\partial_{t_{1}} S_{01}\right)+\xi_{x}\left(\partial_{x_{1}} S_{01}\right)=0 \\
& \xi_{t}\left(\partial_{t_{1}} S_{1 f}\right)+\xi_{x}\left(\partial_{x_{1}} S_{1 f}\right)=0 .
\end{aligned}
$$

This leads to the two relations

$$
\begin{gathered}
\frac{\xi_{t}}{\xi_{x}}=\frac{x_{1}}{t_{1}}, \\
\frac{\xi_{t}}{\xi_{x}}=\frac{x_{1}}{t_{1}-t_{f}} .
\end{gathered}
$$

These conditions, in contrast to Eq. (B3), cannot be fulfilled simultaneously, meaning that there are no lines of constant action in regions with mixed timelike and spacelike contributions. The Fujikawa techniques proposed in this paper cannot be used for such paths.

\section{APPENDIX C: MATHEMATICAL DERIVATION OF EQ. (59)}

Here, we present the mathematical details leading to Eq. (59) in the main text. The integral

$$
\begin{aligned}
K_{S}^{(1)}\left(0, t_{f}\right)= & -\mathcal{N} \cdot \int_{0}^{\infty} d y{\sqrt{y^{2}+1}}^{(d-2)} e^{-m t_{f} y} \\
= & -\mathcal{N} \frac{2^{(d-2) / 3} \pi^{3 / 2}}{\Gamma(1-d / 2)} \\
& \cdot\left(m t_{f}\right)^{\frac{1-d}{2}}\left[\sec \left(\frac{\pi d}{2}\right) \cdot J_{\frac{d-1}{2}}\left(m t_{f}\right)\right. \\
& -2 \csc (\pi d) J_{\frac{d-1}{2}}\left(m t_{f}\right) \\
& \left.+\csc \left(\frac{\pi d}{2}\right) H_{\frac{d-1}{2}}\left(m t_{f}\right)\right]
\end{aligned}
$$

Let us consider the following trigonometric identities:

$$
\begin{aligned}
\sec \left(\frac{\pi d}{2}\right) & =\frac{1}{\cos \left(\frac{\pi d}{2}\right)}=-\frac{1}{\sin \left(\frac{\pi(d-1)}{2}\right)}, \\
\csc \left(\frac{\pi d}{2}\right) & =\frac{1}{\sin \left(\frac{\pi d}{2}\right)}=\frac{1}{\cos \left(\frac{\pi(d-1)}{2}\right)}, \\
\csc (\pi d) & =\frac{1}{2 \sin \left(\frac{\pi d}{2}\right) \cos \left(\frac{\pi d}{2}\right)} \\
& =-\frac{1}{2 \sin \left(\frac{\pi(d-1)}{2}\right) \cos \left(\frac{\pi(d-1)}{2}\right)} .
\end{aligned}
$$

Substituting these identities, we have

$$
\begin{aligned}
& K_{S}^{(1)}\left(0, t_{f}\right) \\
& =\mathcal{N} \frac{2^{(d-2) / 3} \pi^{3 / 2}}{\Gamma(1-d / 2) \cos \left(\frac{\pi(d-1)}{2}\right)} \\
& \cdot\left(m t_{f}\right)^{\frac{1-d}{2}}\left[\frac{\cos \left(\frac{\pi(d-1)}{2}\right) J_{\frac{d-1}{2}}\left(m t_{f}\right)-J_{\frac{1-d}{2}}\left(m t_{f}\right)}{\sin \left(\frac{\pi(d-1)}{2}\right)}-H_{\frac{d-1}{2}}\left(m t_{f}\right)\right] .
\end{aligned}
$$

Finally, using the Bessel function identity

$$
Y_{\alpha}(z)=\frac{J_{\alpha}(z) \cos (\alpha \pi)-J_{-\alpha}(z)}{\sin (\alpha \pi)}
$$

and absorbing all the action-independent constants in the normalization $\mathcal{N}$, we obtain

$$
K_{S}^{(1)}\left(0, t_{f}\right)=\mathcal{N} \cdot\left(m t_{f}\right)^{\frac{1-d}{2}}\left[Y_{\frac{d-1}{2}}\left(m t_{f}\right)-H_{\frac{d-1}{2}}\left(m t_{f}\right)\right] .
$$

[1] G. 't Hooft and M. J. G. Veltman, Ann. Inst. H. Poincare Phys. Theor. A 20, 69 (1974).

[2] O. Lauscher and M. Reuter, Phys. Rev. D 65, 025013 (2001).

[3] M. Reuter and F. Saueressig, Phys. Rev. D 65, 065016 (2002).

[4] D. F. Litim, Phys. Rev. Lett. 92, 201301 (2004).

[5] G. 't Hooft, Commun. Math. Phys. 117, 685 (1988).

[6] A. Ashtekar, V. Husain, C. Rovelli, J. Samuel, and L. Smolin, Classical Quantum Gravity 6, L185 (1989).

[7] S. Carlip, Classical Quantum Gravity 12, 2853 (1995). https://doi.org/10.1088/0264-9381/12/12/005.

[8] C. Teitelboim, Phys. Rev. D 25, 3159 (1982).
[9] M. Henneaux and C. Teitelboim, Ann. Phys. (N.Y.) 143, 127 (1982).

[10] I. H. Redmount and W. M. Suen, Int. J. Mod. Phys. A 08, 1629 (1993).

[11] E. S.Fradkin andD. M. Gitman, Phys. Rev.D44,3230(1991).

[12] H. Kleinert, in Path Integrals in Quantum Mechanics, Statistics, Polymer Physics, and Financial Markets (World Scientific, Singapore, 2006), pp. 1359-1369, ISBN 978-981-4273-55-8.

[13] J. Polchinski, in String Theory (Cambridge University Press, Cambridge, England, 1998), p. 145, ISBN 0521-63303-6. 
[14] T. Padmanabhan, Found. Phys. 25, 11 (1994); 24, 1543 (1994).

[15] L. Brink, S. Deser, B. Zumino, P. Di Vecchia, and P. S. Howe, Phys. Lett. 64B, 435 (1976).

[16] L. Brink, P. Di Vecchia, and P. S. Howe, Nucl. Phys. B118, 76 (1977).

[17] P. Jizba and H. Kleinert, Phys. Rev. E 78, 031122 (2008).

[18] P. Jizba and H. Kleinert, Phys. Rev. D 82, 085016 (2010).

[19] P. Jizba and F. Scardigli, Phys. Rev. D 86, 025029 (2012).

[20] H. Fukutaka and T. Kashiwa, Ann. Phys. (N.Y.) 176, 301 (1987).

[21] T. Padmanabhan, Eur. Phys. J. C 78, 563 (2018).

[22] B. Koch and E. Muñoz, Eur. Phys. J. C 79, 941 (2019).

[23] B. Koch and E. Muñoz, Eur. Phys. J. C 78, 278 (2018).
[24] B. Koch, E. Muñoz, and I. Reyes, Phys. Rev. D 96, 085011 (2017).

[25] L. D. Faddeev and V. N. Popov, Phys. Lett. 25B, 29 (1967).

[26] K. Fujikawa, Phys. Rev. Lett. 42, 1195 (1979).

[27] P. M. Stevenson, Phys. Lett. 100B, 61 (1981).

[28] P. M. Stevenson, Phys. Rev. D 23, 2916 (1981).

[29] P. M. Stevenson, Nucl. Phys. B231, 65 (1984).

[30] B. Koch, P. Rioseco, and C. Contreras, Phys. Rev. D 91 , 025009 (2015).

[31] T. Jacobson and L. S. Schulman, J. Phys. A 17, 375 (1984).

[32] G. N. Ord and D. G. C. Mckeon, Ann. Phys. (N.Y.) 222, 244 (1993).

[33] F. D. T. Smith, Jr., arXiv:quant-ph/9503015.

[34] A. Kull and R. A. Treumann, Int. J. Theor. Phys. 38, 1423 (1999). 